\title{
TECNOLOGÍAS DE LA INFORMACIÓN Y CRECIMIENTO EMPRESARIAL
}

\section{INFORMATION TECHNOLOGIES AND BUSINESS GROWTH}

\author{
William Rodrigo Avendaño Castro' \\ Cesar Augusto Hernández Suarez²
}

Audin Aloiso Gamboa Suárez ${ }^{3}$

\section{RESUMEN}

El presente estudio tiene como objetivo analizar la importancia de las tecnologías de la información en el crecimiento empresarial. Las tecnologías de la información han trastocado todas las esferas de las sociedades, siendo el mundo empresarial una de ellas. Toda organización tiene como prioridad sobrevivir en el mercado, para ello necesita expandirse y crecer, lo cual solo es posible si sostiene elevados estándares de calidad, competitividad y productividad. Las tecnologías de la información facilitan procesos organizativos, productivos y de toma $\overline{1}$ Doctor en Ciencias Sociales y Humanas. Facultad de Ciencias Empresariales. E-mail: williamavendano@ ufps.edu.co, Orcid: https://orcid.org/0000-0002-7510-8222 2 Magister en Educación Matemáticas. Facultad de Educación, Artes y Humanidades. E-mail: cesaraugusto@ ufps.edu.co. Orcid: https://orcid.org/0000-0002-7974-5560 3 Doctor en Ciencias de la Educación por la Universidad de Cartagena. Docente investigador de la Universidad Francisco de Paula Santander. E-mail: audingamboa@ufps. edu.co. Orcid: 0000-0001-9755-6408 de decisiones, posibilitando el crecimiento empresarial a partir de su adecuado uso. Este trabajo es de carácter bibliográfico, teóricodocumental. El contexto actual demanda la expansión empresarial a escalas globales, para lo cual es necesario el uso de nuevas tecnologías que permitan este crecimiento y a su vez el sostenimiento de procesos e intercambios que generen el óptimo funcionamiento organizacional aprovechando al máximo las oportunidades de inversión que facilita el mercado.

PALABRAS CLAVE: Tecnologías de la información, crecimiento empresarial, organizaciones.

\section{ABSTRACT}

The present study aims to analyze the importance of information technologies in business growth. Information technologies have disrupted all 
spheres of societies, the business world being one of them. Every organization's priority is to survive in the market, for this it needs to expand and grow, which is only possible if it maintains high standards of quality, competitiveness and productivity. Information technologies facilitate organizational, productive and decision-making processes, enabling business growth from their proper use. This work is of a bibliographic, theoretical-documentary nature. The current context demands business expansion on a global scale, for which it is necessary to use new technologies that allow this growth and, in turn, the maintenance of processes and exchanges that generate optimal organizational functioning, making the most of the investment opportunities that facilitate the market.

KEYWORDS: Information technology, business growth, organizations.

\section{INTRODUCCIÓN}

El crecimiento empresarial es un elemento que está sujeto a diversos factores y variables, el uso adecuado de las tecnologías de la información es uno de ellos. Es reconocido que las tecnologías de la información (TI) han transformado diversas esferas de las prácticas sociales, una de ella la referida a los negocios y el mundo empresarial.

El uso de nuevas tecnologías suma plusvalía a las instituciones, posibilitando el cambio emergente de los procesos internos de las organizaciones gracias a la actualización constante de múltiples canales y herramientas de trabajo. Este crecimiento nació a mediados de la década de los $60 \mathrm{~s}$ del siglo pasado (Medina-Quintero et al, 2011) y ha incrementado exponencialmente su magnitud e influencia desde entonces, atendiendo las necesidades de los usuarios a la par del incremento del conocimiento, la productividad y la información.

Es incuestionable que el crecimiento empresarial es un objetivo común de todas las organizaciones, las cuales buscan crecer para obtener mayores beneficios que les permita aumentar su sostenibilidad, potenciar su imagen corporativa, construir bienestar organizacional, garantizar su permanencia y consolidación en el mercado.

En este sentido, las TI representan herramientas que son utilizadas a lo interno de la organización para la elaboración de actividades y tareas que permitan optimizar la estrategia de negocios y alcanzar el anhelado crecimiento.

Gracias a las $\mathrm{TI}$, una de las principales estrategias de crecimiento empresarial es la internacionalización de las organizaciones, lo cual es posible gracias a la innovación y el mejoramiento de la productividad que permiten el éxito y la estabilidad organizacional.

En el mundo contemporáneo marcado por la globalización y la inmediatez de la comunicación, la economía mundial ha conducido a las empresas que tienen como horizonte el crecimiento, atiendan la posibilidad de abarcar mercados extranjeros, siendo esta la principal vía de expansión que proporciona el contexto económico y social en la actualidad.

\section{MÉTODO}

La presente investigación se propuso analizar la importancia de las tecnologías de la información en el crecimiento empresarial a partir de un estudio teórico-documental, con diseño bibliográfico y técnica documental, utilizado para evaluar conceptos referentes a la tecnología de la información y el crecimiento económico.

\section{TIPO DE INVESTIGACIÓN}

El tipo de investigación es teórico - documental. Según Arias (2004) es documental, ya que consiste en el estudio de problemas para ampliar el conocimiento apoyado en fuentes bibliográficas y documentales. La originalidad 
se refleja en el enfoque, en los criterios, y en las conclusiones.

La identificación de los textos y documentos permitió seleccionar las unidades de análisis, y, a partir de su lectura, se escogieron los enunciados referidos a los ejes teórico-conceptuales a la tecnología de la información y el crecimiento económico, que se clasificaron en categorías significativas a través de la abstracción.

\section{DISEÑO DE LA INVESTIGACIÓN}

La investigación bibliográfica se caracteriza por la utilización de los datos secundarios como fuente de información, puesto que ésta proporciona el conocimiento de las investigaciones ya existentes -teorías, hipótesis, experimentos, resultados, instrumentos y técnicas usadasacerca del tema o problema a investigar.

Como primera estrategia para la revisión del material teórico documental, se revisa un determinado número de textos relacionados con el tema en estudio, para posteriormente iniciar un proceso de descarte, mediante el cual él se selecciona el material teórico - documental la cual formará parte integrante de la investigación.
Para este caso el material documental o textos seleccionados corresponden a artículos científicos en línea que se encuentran disponibles en bases de datos y/o repositorios de las revistas de acceso abierto (Open Access Journals).

De este modo, se pasa a una etapa de análisis, a través del cual se contrastarán diversas perspectivas de diferentes autores las cuales a su vez presentan posturas contrapuestas sobre las categorías que integran la investigación.

En la presente investigación no se cuenta con muestra, ya que se analizó las fuentes teóricasdocumentales seleccionadas bajo un único momento de recolección dado a la investigación un carácter de transeccional, ya que los datos se recogen en un solo momento al mismo tiempo. Del mismo modo, la investigación no cuenta con un instrumento dado al carácter teórico documental de la misma.

\section{RESULTADOS}

A través del uso del método inductivo, se realizó una revisión y análisis de diferentes artículos científicos relacionados con tecnología de la información y el crecimiento económico de diversos autores (Ver tabla 1) 
Tabla 1. Textos seleccionados sobre los conceptos referentes a la Tecnología de la Información y el Crecimiento Económico.

\begin{tabular}{|c|c|}
\hline & $\begin{array}{l}\text { Andrade \& Campo (2007) Tecnologías de información para la inclusión digital. } \\
\text { Pineda (2008) Las tecnologías de información y comunicaciones (TIC) como fuente de } \\
\text { desarrollo tecnológico. } \\
\text { Medina-Quintero, De la Garza \& Jiménez (2011) Competencia empresarial con el uso de la } \\
\text { información y la tecnología. } \\
\text { Díaz-Batista \& Blanco-Fernández (2018) Adopción y uso de las Tecnologías de la Información } \\
\text { en organizaciones cubanas. } \\
\text { Sánchez \& De la Garza (2018) Tecnologías de información y desempeño organizacional de las } \\
\text { pymes del noreste de México. } \\
\text { Espíndola-Somasa, Fajardo-Ortiz \& Romero (2020). Tecnología de Información y Comunicación } \\
\text { y actividad exportadora en empresas manufactureras en Colombia. } \\
\text { Villegas, Walle-Vazquez \& Álvarez-Aros (2021) Uso de las tecnologías de la información en los } \\
\text { procesos decisorios: un análisis bibliométrico }\end{array}$ \\
\hline $\begin{array}{l}\text { Crecimiento } \\
\text { económico }\end{array}$ & $\begin{array}{l}\text { Gibrat, (1931). Desigualdad económica. } \\
\text { Penrose (1962). Teoría del Crecimiento de la empresa. } \\
\text { De Ravel (1972). El crecimiento de la empresa. } \\
\text { Sallenave (1985). Gerencia y planeación estratégica. } \\
\text { Fernández, García \& Ventura (1988) Análisis del crecimiento sostenible por los distintos } \\
\text { sectores empresariales. } \\
\text { Correa (1999). Factores determinantes del crecimiento empresarial. } \\
\text { Canals (2000). Crecimiento Empresarial: Personas y Tecnología en la Nueva Economía } \\
\text { González \&Acosta (2001). Factores determinantes del crecimiento en la Pyme canaria: influencia } \\
\text { del tamaño, la edad y el sector de actividad. Selección de investigaciones empresariales. } \\
\text { Charan (2004). El crecimiento rentable, un asunto de todos: } 10 \text { herramientas prácticas para el } \\
\text { crecimiento empresarial. } \\
\text { Cardona \& Cano (2005). Territorio, Ciclo de vida y Estructura empresarial: Un puente en la } \\
\text { industrialización regional. } \\
\text { Blázquez, Dorta \& Verona (2006) Concepto, perspectivas y medida del crecimiento empresarial. } \\
\text { Carree \& Thurik (2010) El impacto del espíritu empresarial en el crecimiento económico. } \\
\text { Puerto (2010) La globalización y el crecimiento empresarial a través de estrategias de } \\
\text { internacionalización. } \\
\text { sobre los emprendedores. } \\
\text { Aguilera \& Puerto (2012) Crecimiento empresarial basado en la Responsabilidad Social }\end{array}$ \\
\hline
\end{tabular}


Se parte de la premisa de que el uso de las TI en las organizaciones hace posible el incremento de la eficiencia, con ello un incremento de la satisfacción de usuarios y clientes, posibilitando que dichas organizaciones se vuelvan más competitivas. Sin embargo, estas tecnologías no producen valor por sí mismas, están sujetas a la capacidad de los actores involucrados en disponer de ellas de forma creativa $y$ innovadora para generar el valor deseado.

\section{TECNOLOGÍAS DE LA INFORMACIÓN: VIGENCIA Y ACTUALIDAD}

La sociedad contemporánea se caracteriza por constantesflujos de informacióny conocimientos que permiten intercambios a tiempo real desde los distintos rincones del mundo. El mundo de hoy es global e interconectado en red, cuyas conexiones generan efectos en las distintas esferas: política, economía, deportes, negocios, entre otros. Andrade y Campo (2007) sostienen que la generación, procesamiento y transmisión de información se ha convertido en el principal factor de productividad empresarial.

Toda organización que requiere obtener una ventaja competitiva en el mundo actual requiere de una adecuada implementación de tecnología de punta, lo cual le permite desarrollar capacidades para la atención estratégica del mercado (Sánchez y De La Garza, 2018; Villegas et al, 2021).

Las $\mathrm{TI}$ son todas las formas de tecnología utilizadas para crear, almacenar e intercambiar información en sus diversas formas, por ejemplo, imágenes, datos, audios, videos, presentaciones, documentos, entre otros (Pineda, 2008; Medina-Quintero, 2011). En la actualidad, estas tecnologías representan una ventaja competitiva al ser utilizadas como estrategia de negocio y para los procesos empresariales internos.
La masificación de las TI ha generado niveles de empleos más capacitados, ante lo cual demandan la formación y actualización constante del talento humano. Pineda (2008) sostiene que la aplicación de un gran número de herramientas tecnológicas que los distintos sectores requieren, necesita el desarrollo y la formación de profesionales capacitados para competir adecuadamente en el mercado internacional.

Las $\mathrm{TI}$ han transformado las técnicas y la productividad vinculadas con los procesos organizacionales y administrativas. De igual modo, su uso ha producido cambios sobre la cultura organizacional, las instituciones y la organización social en general, gracias al flujo de información, comunicación y conocimiento (Espíndola-Somasa, 2020).

Gracias a lo anterior, autores como EspíndolaSomasa (2020) se permiten hablar de economía de conocimiento, en la cual una propiedad técnica es capaz de aplicar nuevos conocimientos e informaciones sobre los distintos procesos empresariales, generando de esta forma valor. De este modo, la aplicación adecuada de las técnicas y métodos de producción sobre una tecnología específica se convierte en factor trascendental de cambio y desarrollo económico.

Para Díaz-Batista y Blanco-Fernández (2018), las $\mathrm{TI}$ son utilizadas cada vez más en procesos como la gestión operacional y la toma de decisiones empresariales. Estas herramientas elaboran actividades cada vez más complejas, lo cual permite una creciente influencia en todos los aspectos del desarrollo organizacional. Sobre las empresas, los autores destacan que se evidencian cada vez más su uso en los sistemas de planeación y los sistemas de gestión de la cadena de suministros. Lo anterior 
origina un incremento de la productividad, con ello, aumenta la rentabilidad de la organización gracias al uso adecuado de las TI por parte de sus miembros.

Sin embargo, algunas organizaciones pueden presentar la llamada paradoja de productividad, la cual hace referencia a una relación contradictoria entre las inversiones en $\mathrm{TI}$ que realiza una organización y el desempeño de la misma (Díaz-Batista y BlancoFernández, 2018). Las inversiones de esta naturaleza suelen ser costosas, por lo tanto, es fundamental que la dirigencia y los gerentes atiendan eficientemente su adopción y uso, para evitar pérdidas innecesarias y sacar el máximo provecho posible.

El desarrollo tecnológico, según Pineda (2008), ha impactado en las últimas décadas en generar: 1) acceso masivo al internet de alta velocidad y la conectividad permanente; 2 ) la estratificación y globalización de las audiencias; y, 3) el acceso a distintas fuentes de comunicación e información de forma inmediata.

En un sentido organizacional, las TI permiten a las organizaciones funcionar más allá de la ubicación geográfica y temporal, permitiendo el intercambio a distancia de las informaciones necesarias para el cumplimiento de las labores de la organización y el desarrollo de las actividades necesarias.

Una gestión de calidad está vinculada al uso de herramientas innovadoras, la competitividad empresarial y la capacidad de generar crecimiento organizacional van de la mano con la aplicación adecuada de las $\mathrm{TI}$ y el aprovechamiento máximo de sus ventajas.

\section{INNOVACIÓN Y CRECIMIENTO EMPRESARIAL}

No existe un acuerdo en torno a la definición de crecimiento empresarial. Este corresponde a un concepto bastante diverso, complejo y especulativo. Esta ausencia de definición uniforme ofrece diferentes trabajos que abarcan la noción de crecimiento empresarial desde diversas disciplinas y con ello diversos puntos de vista del mismo fenómeno: sociológico, organizativo, económico, financiero, político, entre otros (Blázquez et al., 2006).

El crecimiento empresarial no cuenta con una teoría general ni una definición conceptual generalmente aceptada (Correa, 1999; Pérez y Acosta, 2001). Sería inadecuado definir de forma homogénea algo que en la propia naturaleza social no lo es. A continuación un recorrido entre las principales nociones de crecimiento económico y algunos de los autores que la discuten.

En primer lugar, Gibrat (1931) apunta que el crecimiento es el resultado de un proceso en el cual coinciden factores internos y externos de la empresa que pone a prueba a la organización de cara a su capacidad para aprovechar las oportunidades de inversión que ofrece el mercado.

Penrose (1962) define el crecimiento empresarial como el resultado caracterizado por la acción compleja de las modificaciones internas que producen un aumento de las dimensiones de la empresa y cambios en sus características, específicamente, la acumulación de capital físico y humano. Este es un proceso, según este autor, que se genera de adentro hacia afuera: la disposición adecuada de los recursos internos permite el potencial posicionamiento externo que ocasiona el crecimiento.

De Ravel (1972) define el crecimiento desde una mirada cuantitativa, vinculando esta idea con el aumento del volumen de producción. Dicho aumento del volumen de producción es posible, precisamente, porque la empresa crece en sus distintas esferas organizativas y de recursos. 
Para Sallenave (1985) el crecimiento es un índice de desempeño económico el cual permite medir la capacidad que tiene la empresa para ampliar su gama de oportunidades comerciales y técnicas.

Por su parte, Fernández, García y Ventura (1988) lo definen como el comportamiento dinámico de la empresa que evidencia el ensanchamiento de sus actividades comerciales, financieras y técnicas en el mercado, caracterizadas por alto dinamismo tecnológico y altas dosis de incertidumbre. Lo anterior obliga a las empresas que deseen asegurar su supervivencia $a$, al menos, igualar a sus rivales más cercanos.

Para Canals (2000) el crecimiento es la expansión que realiza la empresa hacia nuevos mercados, nuevos segmentos de clientela, así como, nuevos espacios geográficos, regiones y países. Este concepto está bastante ligado al boom globalizador de finales de siglo pasado y principios del siglo XXI que resume el éxito empresarial en la capacidad de posicionarse como una marca global.

Por su parte, Charan (2004) asegura que el crecimiento permite a una organización conseguir a los mejores colaboradores, construyendo desde sus capacidades y competencias una fuerte ventaja competitiva.

Cardona y Cano (2005) definen el crecimiento como un proceso intangible que depende de la acumulación de capital físico y humano, dispuestos adecuadamente en la organización y estructura interna de la empresa.

Así mismo, Blázquez et al. (2006) detallan que el crecimiento es un proceso de adaptación exigido por los cambios en el entorno empresarial, en el cual, una organización se compromete a ampliar su capacidad productiva mediante la adquisición de nuevos recursos o el reajuste de sus recursos. Los cambios internos son de carácter organizacional, buscan adecuarse a las modificaciones introducidas, lo que permite sostener la capacidad financiera de la organización y obtener altos estándares de competitividad en el mercado. El crecimiento empresarial se vislumbra como aquel incremento duradero de la dimensión de una unidad económica, manifestado a partir de cambios de estructura y de sistema, que se traducen en progresos económicos variables.

Para Aguilera y Puerto (2012) el crecimiento empresarial es producto del conocimiento del entorno, lo que permite aprovechar al máximo las oportunidades de negocio que éste ofrece.

Varias de estas definiciones se caracterizan por centrar su atención en la importancia del mercado. El crecimiento empresarial, en general, se caracteriza por ser un proceso dinámico que genera cambios necesarios para las empresas, estos cambios pueden ser de tipo cuantitativo: crecimiento financiero, crecimiento productivo, crecimiento de mercado, entre otros. Sin embargo, para obtener tales cambios, las organizaciones requieren características de tipo cualitativo, por ejemplo, los relacionados con la estructura y los mecanismos de dirección y control; estas son las instancias organizacionales que se encargan de impulsar el resto de los cambios (Aguilera \& Puerto, 2012).

El crecimiento empresarial es fundamental para dinamizar las economías y favorecer el desarrollo social y económico de la sociedad (Miralles-Quirós, 2018). Esta es una situación deseable a la cual los líderes de las grandes empresas apuntan como su principal objetivo.

Blázquez et al. (2006), sostienen que el crecimiento empresarial está marcado por diversos factores de índole interna o externa. Entre los factores internos se agrupan la edad, el tamaño, la motivación, la estructura de propiedad y la gestión del conocimiento; mientras que, entre los factores externos, los autores destacan los relativos al contexto (competidores, clientes 
y proveedores) vinculados a la demanda, las mejoras tecnológicas, la accesibilidad a los créditos, el apoyo gubernamental, entre muchos otros. De los factores externos provienen las oportunidades de mercado que dan a la empresa la posibilidad de crecer (Puerto, 2010).

Autores como Carre y Thurik (2010) y Galindo y Méndez (2011) trazan una relación directa entre crecimiento económico y la capacidad de asumir riesgos por parte de la organización. Tener un crecimiento sostenido implica la capacidad de una empresa para, en primer lugar, identificar oportunidades de inversión en un entorno incierto; $y$, en segundo lugar, arriesgar constantemente su capital en aras de producir bienes y servicios eficientemente, contribuyendo al aumento de su productividad.

En el mundo contemporáneo, las empresas que no aspiren a posicionar sus productos o marcas en el extranjero limitarán su crecimiento y quedarán expuestas en el mediano plazo a las limitaciones económicas de un mercado local (Puerto, 2010). Para ello, es clave la utilización adecuada de las nuevas tecnologías de la información.

\section{IMPORTANCIA DE LAS TECNOLOGÍAS DE LA INFORMACIÓN EN EL CRECIMIENTO EMPRESARIAL}

Ante la irrupción de nuevas tecnologías, las empresas se ven en la necesidad de evaluar su adopción en busca de la obtención de beneficios y del incremento de la eficiencia. Las tecnologías permiten reducir en nivel de incertidumbre en medio de un contexto de cambio constante.

Estos cambios globales inducen que la tecnología llegue inevitablemente a todos los rincones de la administración empresarial. La gerencia, ante la infinita diversidad de soluciones tecnológicas, debe generar procesos de evaluación exhaustivos y toma de decisiones acorde con sus capacidades y necesidades, en aras de integrar los nuevos recursos con sus distintos ámbitos de operaciones y sus diversas realidades.

La introducción de las tecnologías de la información en entornos empresariales permite la digitalización de procesos administrativos y comunicativos, facilitando la flexibilidad del entorno organizacional. Para que su aplicación sea óptima resulta indispensable contar con personal competente y capacitado, por lo tanto, es importante entender que la introducción de herramientas tecnológicas debe adaptarse a cada contexto particular y a las condiciones específicas de cada empresa.

Esta capacidad de adaptación que ganan las empresas con la introducción de tecnologías genera un impacto efectivo sobre las decisiones estratégicas a nivel organizacional. Las $\mathrm{TI}$ modifican el entorno, las tareas, las condiciones, la estructura, la relación con el público, la relación con los empleados, y más.

La expansión de las TI y su capacidad de filtrarse en todos los estratos de la sociedad generan aplicaciones cada vez más amplias para estas herramientas, permitiendo interconectar con altos estándares de calidad a prácticamente todos los sectores de una organización.

Los distintos equipos de trabajo pueden conectarse a través de videoconferencias desde cualquier lugar en cualquier momento, desde los niveles más operativos hasta los directivos, permitiendo nuevas posibilidades comunicativas y decisorias que facilitan la eficacia de la organización y con ello las respuestas acertadas para atender las demandas de su entorno.

Así mismo, la capacidad de digitalizar todos los procesos y la información permite una mayor influencia y capacidad de manejo sobre los procesos de la organización, gracias a los formatos de audio, video y texto, un sujeto posee una amplia masa de información la cual 
puede llevar de un rincón a otro del mundo en cuestión de segundos. Estas facilidades suponen ventajas cuantitativas y cualitativas para el manejo organizacional y la capacidad de expansión de las empresas.

La implementación de las TI genera la reducción de costos operativos y administrativos, así como también, el aumento de la calidad de los servicios, productos y procesos, con lo cual las empresas ven favorecidas su productividad y competitividad.

De este modo, las $\mathrm{TI}$ son una herramienta poderosa e imprescindibles para aprovechar las oportunidades que presenta el mercado y obtener la ventaja competitiva que toda organización desea en aras de su crecimiento. En la actualidad las $\mathrm{TI}$ son un estándar de calidad en toda gran empresa, convirtiéndose en herramientas de interés, siendo utilizadas de manera constante y dinámica en la realización de tareas y para la mejora de la productividad y de la competitividad.

Las organizaciones que aspiran a crecer, se apoyan en las $\mathrm{TI}$ como recurso fundamental para potenciar su rendimiento e incrementar sus niveles de eficiencia. Este recurso permite sostener la estrategia empresarial en la realización óptima y veloz de las tareas diarias de la organización, facilitando así el incremento del desempeño y fortaleciendo la competitividad. De este modo, las TI se convierten en un recurso estratégico clave para aprovechar las oportunidades de expansión hacia nuevos mercados, además de posibilitar el funcionamiento y el comercio electrónico, permiten, gracias a la disminución de costos y aumento de productividad, incrementar las probabilidades de éxito de la empresa.

\section{REFLEXIONES FINALES}

La posibilidad de implementar tecnología es distinta para cada empresa. Así como las oportunidades que brinda el entorno son distintas para cada organización, es distinta la capacidad de aprovecharlas también. Al momento de evaluar la adquisición y puesta en funcionamiento de nuevas herramientas, las organizaciones deben considerar varios factores, entre ellos, el costo de aplicación, las demandas del entorno y el comportamiento de las empresas rivales.

La postergación de implementación de nuevas tecnologías retrasa la obtención de beneficios y aleja las posibilidades de incrementar la productividad, competitividad y el crecimiento para la empresa. De este modo, la relación costo-beneficio resulta fundamental al momento de evaluar la adquisición de nuevas tecnologías.

El uso de las tecnologías por sí solas no garantiza un beneficio empresarial. Es importante que el talento humano este capacitado adecuadamente para su uso, al mismo tiempo, es clave que la organización posea una estrategia bien definida y esclarecida que permita sacarle el máximo provecho a las herramientas.

Las empresas y organizaciones aspiran naturalmente a su supervivencia, para ello es fundamental construir un crecimiento sostenido apoyado en el uso de las tecnologías de la información que favorezcan un mejor desempeño organizacional, un aumento de la productividad y fortalecimiento de la competitividad en el mercado global. 


\section{REFERENCIAS BIBLIOGRÁFICAS}

Aguilera, A., \& Puerto, D. (2012) Crecimiento empresarial basado en la Responsabilidad Social. Pensamiento \& Gestión, (32), 1-26. http://www.redalyc.org/articulo. oa?id=64623932002

Andrade, J., \& Campo, M. (2007) Tecnologías de información para la inclusión digital. Apertura, 7(6), 63-75. http://www.redalyc. org/articulo.oa?id=68800606

Blázquez, F., Dorta, J., \& Verona, M. (2006) Concepto, perspectivas y medida del crecimiento empresarial. Cuadernos de Administración, 19(31), 165-195. http:// www.redalyc.org/articulo.oa?id=20503107

Canals, J. (2000). Crecimiento Empresarial: Personas y Tecnología en la Nueva Economía. Revista Empresa y Humanismo, 2(2), 341-350.

Cardona, M., \& Cano, C. (2005). Territorio, Ciclo de vida y Estructura empresarial: Un puente en la industrialización regional. Fondo Editorial Universidad EAFIT.

Carree, M., \& Thurik, R. (2010) The impact of entrepreneurship on economic growth. En Z.J. Acs \& D.B. Audretsch. (Eds.), Handbook of Entrepreneurship Research (pp. 557-594). Springer Science Business Media.

Charan, R. (2004). El crecimiento rentable, un asunto de todos: 10 herramientas prácticas para el crecimiento empresarial. Empresa Activa.

Correa, A. (1999) Factores determinantes del crecimiento empresarial (Tesis doctoral, Universidad de la Laguna). Repositorio institucional RIULL. https://riull.ull.es/xmlui/ handle/915/10016
De Ravel, R. (1972). El crecimiento de la empresa. Guadiana.

Díaz-Batista, J., \& Blanco-Fernández, Y. (2018) Adopción y uso de las Tecnologías de la Información en organizaciones cubanas. Ingeniería Industrial, 39(3), 273-282. https://www.redalyc.org/articulo. oa?id=360458817006

Espíndola-Somasa, L., Fajardo-Ortiz, E., \& Romero, H. (2020) Tecnología de Información y Comunicación y actividad exportadora en empresas manufactureras en Colombia. Revista Venezolana de Gerencia (RVG), 25(4), 377-394.

Fernández, A., García, R., \& Ventura, J. (1988) Análisis del crecimiento sostenible por los distintos sectores empresariales. Documento de trabajo de la Universidad de Oviedo, (3), 1-27.

Galindo, M., \& Méndez, M. (2011) La actividad emprendedora y competitividad: Factores que inciden sobre los emprendedores. Papeles de Europa, (22), 61-75.

Gibrat, R. (1931). Les inegalites economiques. Librairie du Recueil Sirey.

González, A., \& Acosta, M. (2001). Factores determinantes del crecimiento en la Pyme canaria: influencia del tamaño, la edad y el sector de actividad. En Selección de investigaciones empresariales. Convocatoria 2000: Programa de Fomento a la Investigación Empresarial en las Universidades Canarias del la Fundación Fyde-Cajacanarias y la Consejería de Economía, Hacienda y Comercio (pp. 75112). Fundación FYDECajaCanarias. 
Medina-Quintero, J., De la Garza, M., \& Jiménez, K. (2011) Competencia empresarial con el uso de la información y la tecnología. Investigación Administrativa, (108), 7-17. $\quad$ http://www.redalyc.org/articulo. oa?id=456045339001

Penrose, E. (1962). Teoría del Crecimiento de la empresa. Ediciones Aguilar.

Pineda, L. (2008) Las tecnologías de información y comunicaciones (TICs) como fuente de desarrollo tecnológico. Universidad \& Empresa, 7(15), 137-149. http://www. redalyc.org/articulo.oa?id=187214457006

Puerto, D. (2010) La globalización y el crecimiento empresarial a través de estrategias de internacionalización. Pensamiento \& Gestión, (28), 171-195. http://www.redalyc. org/articulo.oa?id=64615176009

Sallenave, J. (1985). Gerencia y planeación estratégica. Norma.

Sánchez, M., De la Garza, M. (2018). Tecnologías de información y desempeño organizacional de las pymes del noreste de México. Revista Venezolana de Gerencia (RVG), 23(82), $298-313$.

Villegas, L., Walle-Vazquez, M., \& Alvarez-Aros, E. (2021) Uso de las tecnologías de la información en los procesos decisorios: un análisis bibliométrico. Revista Venezolana de Gerencia (RVG), 26(93), 92-109. 